\title{
Estrategias de afrontamiento y estilos parentales en madres de niños con y sin trastorno por déficit de atención e hiperactividad
}

\author{
Rocío González ${ }^{\star}$ Liliana Bakker ${ }^{\star *}$, Josefina Rubiales ${ }^{\star * *}$
}

\begin{abstract}
Resumen
El objetivo del presente estudio consiste en comparar las estrategias de afrontamiento y el estilo parental de madres de niños con y sin diagnóstico de trastorno por déficit de atención e hiperactividad (TDAH). La muestra se conformó en dos grupos: un grupo de madres con un hijo con diagnóstico de TDAH $(N=14)$ y un grupo control $(N=35)$. El abordaje metodológico se realizó por medio de la aplicación de dos escalas que evalúan estilos educativos parentales y del Cuestionario de Afrontamiento del Estrés (CAE) diseñado para evaluar siete estilos básicos de afrontamiento. Los resultados muestran diferencias en la percepción de los estilos parentales de las madres de niños con TDAH en comparación con el grupo control, manifestando mayor crítica, menor expresión afectiva y comportamientos educativos más rígidos. Si bien no se observan diferencias significativas entre los grupos en cuanto a las estrategias de afrontamiento utilizadas, sí se evidencia una tendencia a utilizar en mayor medida estrategias de afrontamiento racional y emocional en madres de niños con TDAH. Identificar el estilo parental más funcional y las estrategias de afrontamiento al estrés más efectivas para estas madres tiene una implicación directa sobre el tratamiento del trastorno ya que podrían jugar un rol importante en el curso de la enfermedad de su hijo.
\end{abstract}

Palabras clave: estilo parental, estrategias de afrontamiento, madres, niños, TDAH.

Coping Strategies and Parenting Styles in Mothers of Children with and without Attention Deficit Hyperactivity Disorder

\begin{abstract}
The aim of this study was to compare coping strategies and the parenting styles of mothers of children who have and have not been diagnosed with Attention Deficit Hyperactivity Disorder (ADHD). The sample consisted of two groups: one of mothers of a child diagnosed with ADHD $(N=14)$ and a control group $(N=35)$. The methodological approach was to apply two scales that evaluate parental educational styles and the Coping Strategies Questionnaire (CSQ), designed to evaluate seven basic coping styles. The results show differences in the perception of parenting styles of mothers of children with ADHD compared to the control group, taking the form of more criticism, less expression of affection and more rigid educational behavior. While there were no significant differences between groups in terms of the coping strategies used, a tendency could be seen among mothers of children with ADHD to rely more on rational and emotional coping strategies. Identifying the most effective parenting style and coping strategies has direct implications for the treatment of this disorder, as these could have an important impact on how a child's illness develops.
\end{abstract}

Keywords: parenting style, coping strategies, mothers, children, ADHD.

\section{Estratégias de enfrentamento e estilos parentais em mães de criança com e sem transtorno por déficit de atenção e hiperatividade}

\section{Resumo}

O objetivo do presente artigo consiste em comparar as estratégias de enfrentamento e o estilo parental de mães de criança com e sem diagnóstico de transtorno por déficit de atenção e hiperatividade (TDAH). A amostra esteve conformada por dois grupos: um grupo de mães com um filho com diagnóstico de TDAH $(N=14)$ e um grupo controle $(N=35)$. A abordagem metodológica foi realizada por meio da aplicação de duas escalas que avaliam estilos educativos parentais e do Questionário de Enfrentamento do Estresse (QEE) desenhado para avaliar sete estilos básicos de enfrentamento. Os resultados mostram diferenças na percepção dos estilos parentais das mães com criança com TDAH em comparação com o grupo controle, manifestando maior crítica, menor expressão afetiva e comportamentos educativos mais rígidos. Embora não tenham sido observadas diferenças significativas entre os grupos quanto às estratégias de enfrentamento utilizadas, evidencia-se uma tendência a utilizar em maior proporção estratégias de enfrentamento racial e emocional em mães de criança com TDAH. Identificar o estilo parental mais funcional e as estratégias de enfrentamento do estresse mais efetivas para essas mães tem uma implicação direta sobre o tratamento do transtorno, já que poderiam desempenhar um papel importante no curso da doença de seu filho.

Palavras-chave: estilo parental, estratégias de enfrentamento, mães, criança, TDAH. 


\section{Introducción}

El trastorno por déficit de atención con hiperactividad (TDAH) es el trastorno del desarrollo neurológico en edad pediátrica más frecuente, se inicia antes de los 12 años de edad y puede persistir a lo largo de la vida, es decir, es de carácter crónico (López-Martín, Albert, Fernández-Jaén y Carretié, 2010; Ghuman y Ghuman, 2014). Aproximadamente del 50 al $70 \%$ de los niños diagnosticados con TDAH continúan con síntomas del trastorno en la adolescencia y un 40 al 50\% en la etapa adulta, con síntomas severos en el 10 al 15\% de los casos (Ortiz-Léon y Jaimes-Medrano, 2007). Actualmente es definido como un patrón persistente de síntomas de inatención o hiperactividad-impulsividad que es más frecuente y grave que el observado habitualmente en las personas con un grado de desarrollo similar (American Psychiatric Association [APA], 2013).

Aunque la etiología no ha sido completamente aclarada, es considerado un trastorno con un tipo de herencia poligénica multifactorial, en la que se presenta una compleja combinación de factores genéticos, biológicos y ambientales. Entre los factores ambientales parecen encontrarse las circunstancias perinatales y los métodos de crianza y educación (Catelan-Mainardes, 2010; Etchepareborda, Díaz-Lucero y de Ramón, 2011; Sadek, 2014).

Precisamente, se ha evidenciado que la calidad de las relaciones familiares y los patrones de interacción podrían estar relacionados con el desarrollo y curso de diversos patrones de psicopatología de la niñez (Montiel-Nava, Montiel-Barbero y Peña, 2005), por lo que se considera que el ambiente psicosocial desempeña un papel modulador fundamental sobre la predisposición biológica al TDAH, influyendo en la forma en la que la hiperactividad, la impulsividad y la inatención son comprendidas y manejadas por la familia (Miranda-Casas, Grau-Sevilla, Marco-Taberner y Roselló, 2007). Por consiguiente, si bien estas variables ambientales no son factores causales, sí modulan la evolución del trastorno y pueden considerarse como variables de riesgo si aumentan la probabilidad de aparición de sintomatologías, o como variables de protección si disminuyen o amortiguan el impacto de los acontecimientos de riesgo (Grau-Sevilla, 2007).

Los síntomas tienen un gran impacto en el desarrollo del individuo e interfieren en su funcionamiento social, emocional, conductual y cognitivo, ocasionando una importante disfuncionalidad no sólo en el niño, sino también en su familia y en el grupo de compañeros (Cardo y Servera, 2008).
Diversos estudios han mostrado el impacto que tiene el TDAH sobre la dinámica familiar - en lo referente tanto al estrés parental que conlleva- así como sobre los estilos parentales de crianza característicos de esta población. En este sentido, se ha observado que la disfuncionalidad familiar es mayor para aquellas familias de niños con TDAH que para las familias de niños sin el trastorno, y está consistentemente asociada con las alteraciones conductuales, la gravedad y la persistencia de los síntomas en los niños (Montiel-Nava et al., 2005; Miranda-Casas et al., 2007; Yousefia, Far y Abdolahian, 2011).

Si bien existen múltiples variables del contexto familiar que se han estudiado como posibles factores influyentes (nivel socioeconómico, orden y número de hermanos, personalidad y sintomatología parental, tipo familiar, inconsistencia parental; Raya-Trenas, Herreruzo-Cabrera y Pino-Osuna, 2008), este estudio se centra en analizar dos variables ambientales que forman parte de lo que se entiende por ambiente psicosocial familiar en niños con TDAH: el estilo educativo parental y las estrategias de afrontamiento ante situaciones de estrés.

En lo referente a los estilos parentales, estos se describen como tendencias globales de comportamiento, siendo el resultado de la interacción de diferentes actitudes y conductas de los padres a la hora de interactuar con el niño, influyendo sobre su comportamiento (Torío-López, Peña-Calvo y Rodríguez-Menéndez, 2008). Dentro de este ámbito de investigación, el modelo de Baumrind (1996) define el estilo parental como una característica de la relación entre padres e hijos más que como una característica de los padres, entendiendo estas relaciones desde un enfoque bidireccional (Bersabé, Fuentes y Motrico, 2001). Baumrind tiene en cuenta las interacciones que se enmarcan dentro de dos dimensiones: afecto-comunicación y control-exigencias, lo cual dará como resultado tres estilos: autoritario, permisivo y democrático (López-Soler, Puerto, López-Pina y Prieto, 2009; Baumrind, Larzelere y Owens, 2010).

El estilo autoritario se manifiesta en una tendencia a utilizar medidas de castigo o de fuerza para controlar el comportamiento de los hijos con patrones rígidos preestablecidos. Por su parte, los padres con estilo permisivo evitan el recurso a la autoridad, el uso de las restricciones y los castigos proporcionando autonomía, aunque a expensas de la falta de límites. Por último, los padres con estilo democrático tienden a dirigir racionalmente las actividades del niño imponiéndole roles y conductas maduras utilizando el razonamiento y la negociación. Es un estilo que se caracteriza por 
la comunicación bidireccional y un énfasis compartido entre la responsabilidad social de las acciones y el desarrollo de la autonomía e independencia en el hijo (Baumrind, 1996).

Actualmente existen evidencias sobre las diferencias de género referentes al estilo parental. En este sentido, se ha evidenciado que las madres tienden a mostrar prácticas parentales más cercanas al estilo autoritativo o democrático, mientras que los padres muestran prácticas más cercanas al estilo autoritario o rígido (Russel, Hart, Robinson y Olsen, 2003). Si bien se considera que los padres son activos en el proceso de socialización y difieren en su estilo de crianza en relación con las madres (Raya-Trenas et al., 2008), en este trabajo se limitará al análisis del estilo materno ya que, por un lado, se considera que son estas generalmente quienes se encargan de supervisar las costumbres y actividades de la vida cotidiana del niño, y por otro, porque el interés del presente trabajo reside en comparar su estilo con el de madres de hijos sin el trastorno, dejando para futuras investigaciones el análisis del estilo paterno.

En este sentido, las relaciones entre madres y niños con TDAH suelen marcarse por el conflicto, ciertamente porque estos son menos dóciles, menos sumisos y solicitan más estimulación y ayuda que otros niños (Roselló, García-Castellar, Tárraga-Mínguez y Mulas, 2003). La crianza de un niño con TDAH ofrece numerosos retos y las madres usualmente reportan altos niveles de frustración en sus intentos de manejar y controlar su conducta. Esto sucede entre otras cosas porque los métodos de disciplina usuales no funcionan con la misma eficacia que con otros niños debido a que poseen mayores dificultades para obedecer las órdenes e inhibir las respuestas impulsivas, y son menos sensibles a las peticiones de los adultos, por lo que su estilo personal provoca procedimientos de disciplina más inconsistentes y coercitivos en las madres. Asimismo, estas conductas de los niños desencadenan cogniciones negativas sobre su rol de madres, baja autoestima, frustración, aislamiento social y sentimientos de culpa (Miranda, Presentación, Colomer y Roselló, 2011). Esta situación repercute a su vez en el propio niño, en su desarrollo emocional y en su autoestima, promoviendo un círculo vicioso de interacciones negativas y sentimientos de fracaso que perpetuarán las dificultades familiares y las manifestaciones sintomáticas del TDAH (Grau-Sevilla, 2007; Alizadeh, Applequist y Coolidge, 2007).

Se ha evidenciado además que las madres de niños con TDAH presentan frecuentemente dos estilos: autoritarios o permisivos; el primero, ya que, al experimentar mayor estrés, utilizan más órdenes y pautas, muestran más desaprobación, más castigos físicos, manejan menos efectivamente el comportamiento de sus hijos y exhiben más comportamientos negativos que las madres de niños sin TDAH. Así, habría dos tipos de estilos parentales negativos que se relacionan con actuales y futuros problemas de conducta en los niños: el estilo parental autoritario y el permisivo (McKee, Harvey, Danforth, Ulaszek y Friedman, 2004). Estudios como el realizado por Goldstein, Harvey y Friedman-Weieneth (2007) han encontrado una significativa relación entre el estilo parental autoritario y el TDAH, en el que las madres presentan niveles elevados de estrés mostrándose más críticas, controladoras y autoritarias, con menor inclinación a resolver problemas (Alizadeh y Andries, 2002; Raya-Trenas et al., 2008; Yousefia et al., 2011). Asimismo, se ha evidenciado que estas conductas parentales disfuncionales e interacciones negativas están asociadas con altos niveles de estrés parental (Olhaberry y Farkas, 2012).

En relación con ello, diversas investigaciones muestran que el nivel de estrés parental es más elevado en madres de niños con problemas en el desarrollo que en madres de niños sin estos problemas, lo cual afecta a su vez la conducta educativa parental (Tomanik, Harris y Hawkins, 2004; Long, Gurka y Blackman, 2008; López, Clifford, Minne y Ouellette-Kuntz, 2008; Dabrowska y Pisula, 2010). En un estudio reciente se evidenció que tener un hijo con problemas de conducta predice el $57 \%$ de la variancia del estrés parental (Solem, Christophersen y Martinussen, 2011). Las mayores fuentes de estrés que experimentan las madres de niños con TDAH son la permanencia de la condición (cronicidad), la desaprobación familiar, escolar y social que genera la conducta disfuncional de sus hijos y, en algunos casos, el insuficiente apoyo profesional ( $\mathrm{Da}$ browska y Pisula, 2010).

Estudios sobre estrés parental en madres de niños con TDAH muestran que ellas presentan poco apoyo social y tienden a utilizar más estrategias de afrontamiento desadaptativas que las madres de niños sin el trastorno (Siegel y Keefe, 2007; Olhaberry y Farkas, 2012), más aún, presentan niveles de estrés más elevado que los padres de niños con TDAH (Narkunam, Hashim, Sachdev, Pillai y Ng, 2014).

El Modelo Procesual de Estrés de Lazarus y Folkman (1984) conceptualiza el estrés como una interpretación o evaluación subjetiva tanto de la existencia de un potencial estresor como de la inexistencia de habilidades o recursos para afrontarlo. Así, los individuos no sólo perciben de forma diferente las amenazas de las 
situaciones estresantes, sino que también emplean diferentes habilidades, capacidades y recursos, tanto personales como sociales, para hacer frente a las condiciones potencialmente estresoras (Cockerham, 2001; Sandín, 2003; Verduzco-Alvarez-Icaza, Lucio-Gómez-Maqueo y Durán-Patiño, 2004). Para explicar las diferencias que existen entre los distintos individuos en la adaptación al estrés, se ha investigado la influencia de las estrategias de afrontamiento como mediadoras de los efectos de los estresores sobre la respuesta (Sandín, 2003; Mikulic y Crespi, 2008).

En este sentido, el concepto de afrontamiento del estrés hace referencia a los esfuerzos conductuales y cognitivos que se llevan a cabo para hacer frente a las demandas externas o internas generadoras del estrés, que son evaluadas como algo que excede los recursos personales. Estas estrategias pueden ser adaptativas o no (Lazarus y Folkman, 1984; Lazarus, 1993), y dependiendo de la efectividad de la implementación de la estrategia, si el individuo tiene éxito para solucionar la situación problemática presente, tenderá a repetirla ante situaciones similares, mientras que en caso contrario buscará otro recurso (Olson y McCubbin, 1989).

Existen numerosas clasificaciones sobre las estrategias de afrontamiento; sin embargo, más allá de su número, se han diferenciado tres dimensiones: según el método, la focalización y la actividad. En la dimensión según el método, los estilos de afrontamiento pueden ser activos o de control directo, pasivos o de control indirecto, y de evitación o abandono de control; según la focalización puede ser dirigido al problema, a la emoción o a modificar la evaluación inicial de la situación, y según la actividad, puede ser cognitivo o conductual (Folkman y Lazarus, 1984; Verduzco-Alvarez-Icaza et al., 2004; Mikulic y Crespi, 2008).

En el caso de las madres de niños con TDAH, se presentan datos insuficientes y contradictorios acerca del uso y la eficacia en la implementación de estrategias de afrontamiento ante situaciones de estrés. Por un lado, se ha evidenciado que estas madres tienden a utilizar más estrategias de afrontamiento desadaptativas que las madres de niños sin el trastorno (Siegel y Keefe, 2007; Durkan, Erdem y Tufan, 2008), sumado a la tendencia a presentar mayor inconsistencia y hostilidad parental, menos calidez y mayores niveles de depresión y ansiedad (Cussen, Sciberras, Ukoumunne y Efron, 2012). Por otro lado, los resultados de un estudio reciente muestran que si bien las madres de niños sin TDAH reportan un uso mayor de estrategias basadas en la resolución planificada de problemas, no se evidencian diferencias significativas en la utilización de otro tipo de estrategias de afrontamiento entre madres de niños con y sin TDAH (Tajalli, Hooman, Afrooz y Bonab, 2011).

Si bien se han desarrollado diversos estudios de investigación tendientes a indagar el estrés parental y los estilos educativos parentales (Pimentel, Vieira-Santos, Santos y Vale, 2011; Graziano, McNamara, Geffken y Reid, 2011; Tan, Camras, Deng, Zhang y Lu, 2012; Park y Walton-Moss, 2012), las relaciones existentes entre el estilo parental y las estrategias de afrontamiento han sido escasamente estudiadas, ya que en su mayoría se han centrado en las estrategias de afrontamiento de los niños (Richaud, 2005).

Una aproximación en este sentido es el estudio realizado por Henao-López (2008), el cual estuvo orientado a proporcionar evidencia sobre las posibles conexiones entre la estructura de personalidad paternal y maternal en términos del perfil cognitivo parental (estrategias de afrontamiento y esquemas maladaptativos) y el estilo parental específico en torno a la crianza de los hijos. Aunque los resultados no permitieron establecer relaciones contundentes y firmes sino tendencias, sí se evidenciaron relaciones entre el estilo autoritario materno y las estrategias de afrontamiento tendientes a la búsqueda de apoyo, expresión de la dificultad de afrontamiento y negación; entre el estilo permisivo materno y la estrategia de espera, expresión de la dificultad de afrontamiento y negación; y entre el estilo democrático materno y la estrategia de solución de problemas, reevaluación positiva de la situación y autonomía. Sin embargo, no se han encontrado estudios en esta temática realizados en familias de niños con TDAH.

Por ello, el objetivo de este trabajo es analizar y comparar dos variables ambientales familiares en niños con TDAH: el estilo educativo parental de la madre y sus estrategias de afrontamiento ante situaciones de estrés.

\section{Métodos}

\section{Diseño}

En el presente estudio se empleó un diseño ex post facto retrospectivo con dos grupos, uno cuasi control, según la clasificación de Montero y León (2007).

\section{Participantes}

La muestra estuvo compuesta de manera intencional por 49 participantes divididos en dos grupos. Un grupo 
estuvo constituido por 14 madres (media de edad: 38,9; desviación estándar: 1,73) de niños con diagnóstico de TDAH, los cuales fueron diagnosticados clínicamente por médicos neurólogos infantiles pertenecientes a Centros de Salud de la ciudad de Mar del Plata, Argentina, siguiendo los criterios diagnósticos para el TDAH según el DSM-IV TR (APA, 2002). Asimismo, el diagnóstico de los niños fue confirmado utilizando la Escala SNAP IV versión adaptada a los criterios del DSM IV para padres y docentes (Grañana et al., 2006), el listado de síntomas Child Behavior Checklist (CBCL; Samaniego, 1998) y el Test de Inteligencia de Weschler (wISCIII) para niños y adolescentes (Wechsler, 1994). Por tanto, los niños diagnosticados clínicamente por médicos neurólogos infantiles debían cumplir los siguientes criterios para ser incluidos en la muestra: presentar valores por encima del punto de corte esperado en la escala SNAP específica de TDAH (Inatención maestros: 2,56; Inatención padres: 1,78; Hiperactividad-impulsividad maestros: 1,78; Hiperactividad-impulsividad padres: 1,44; Combinado maestros: 2,00; Combinado padres: 1,67); presentar valores por encima del punto de corte esperado (т: 70) en la dimensión problemas de atención del listado de síntomas СвСL; presentar un nivel intelectual promedio (CI mayor a 90) y no estar actualmente en tratamiento. Los resultados de estas pruebas, junto con la entrevista a los padres y la evaluación neuropsicológica a los niños, permitió confirmar el diagnóstico de los niños. El grupo control se conformó por 35 madres (media de edad: 35,8 ; desviación estándar: 1,96 ) de niños sin diagnóstico de TDAH, los cuales presentaban valores por debajo del punto de corte en la escala SNAP y en la CBCL de padres y maestros. Los niños de ambos grupos tenían edades comprendidas entre 8 y 12 años, eran niños escolarizados residentes en la ciudad, presentaban un ci promedio y no tenían antecedentes de enfermedades neurológicas o psiquiátricas, trastornos del aprendizaje ni trastorno de nivel intelectual.

\section{Instrumentos}

El abordaje metodológico se realizó por medio de la aplicación de dos instrumentos. Para evaluar estilos educativos parentales se utilizaron dos escalas (Afecto $\mathrm{y}$ Normas-Exigencias) diseñadas y validadas por Bersabé et al. (2001).

La escala de Afecto consta de dos factores: afecto/comunicación y crítica/rechazo; esta posee una fiabilidad con consistencia interna alta (Factor I-padre $\alpha=0,90$; Factor II-padre $\alpha=0,83$; Factor I-madre $\alpha=0,87$; Factor II-madre $\alpha=0,81$ ). Además, los índices de homogeneidad de los ítems (correlación ítem-puntuación total corregida) se encuentran por encima de 0,45 , lo que indica un buen comportamiento psicométrico.

La escala de Normas y Exigencias se divide en tres factores de acuerdo con la forma de establecer y exigir el cumplimiento de las normas: forma inductiva (democrática), rígida (autoritaria) e indulgente (permisiva). En cada factor, la fiabilidad con una consistencia interna también es elevada (Factor I-padre $\alpha=0,85$; Factor II-padre $\alpha=0,73$; Factor III-padre $\alpha=0,60$; Factor I-madre $\alpha=0,80$; Factor II-madre $\alpha=0,72$; Factor III-madre $\alpha=0,64)$. Los índices de homogeneidad de los ítems se encuentran por encima de 0,40, lo que indica un buen comportamiento psicométrico.

Las dos escalas se corresponden con cuestionarios tipo Likert, en los cuales el participante expresa el grado de acuerdo o desacuerdo con cada afirmación seleccionando una alternativa de respuesta (Nunca, Pocas veces, Algunas veces, Muchas veces, Siempre), marcando con una cruz $(\mathrm{x})$ la opción que más se acerque a su forma de pensar. La Escala de Afecto/Comunicación (EA) y la Escala de Normas y Exigencias (ENE) constan de 20 y 28 ítems respectivamente. La puntuación de la escala se obtiene sumando los valores de las respuestas individuales, lo que proporciona una puntuación total para cada factor.

Asimismo, se utilizó el Cuestionario de Afrontamiento del Estrés (CAE) diseñado por Sandin y Chorot (2003). Dicho cuestionario consiste en una medida de autoinforme que evalúa siete estilos básicos de afrontamiento: focalizado en la solución del problema (FSP), autofocalización negativa (AFN), reevaluación positiva (REP), expresión emocional abierta (EEA), evitación (EVT), búsqueda de apoyo social (BAS) y religión (RLG). Los coeficientes de fiabilidad de Cronbach para las siete subescalas varían entre 0,64 y $0,92(\mathrm{M}=0,79)$. El análisis factorial evidencia una estructura de dos factores: el primer factor es equivalente a la dimensión de afrontamiento racional, focalizado en el problema (Folkman y Lazarus, 1988) o afrontamiento activo (Billings y Moos, 1981), que incluye las siguientes subescalas: FSP, REP y BAS; la segunda dimensión coincide con la dimensión de afrontamiento centrada en la emoción (Folkman y Lazarus, 1988) o con el afrontamiento pasivo (Billings y Moos, 1981), que incluye las siguientes subescalas: AFN Y EEA.

El cuestionario es de tipo Likert, en el cual el participante expresa el grado de acuerdo o desacuerdo 
con cada afirmación seleccionando una alternativa de respuesta (Nunca, Pocas veces, Algunas veces, Frecuentemente, Siempre), marcando con una cruz (x) la opción que más se acerque a su forma de actuar en aquellas situaciones de estrés que recientemente ha tenido que afrontar (aproximadamente durante el pasado mes). La escala consta de 42 ítems y la puntuación se obtiene sumando los valores de las respuestas individuales, lo cual proporciona una puntuación total para cada estilo básico de afrontamiento. Adicionalmente, se obtienen medidas más generales del afrontamiento, correspondiente a dos subescalas generales: la subescala de afrontamiento racional (AR) con predomino de focalización en el problema y la subescala de afrontamiento emocional (AE).

\section{Procedimiento}

El presente estudio se realizó como parte del proyecto mayor del grupo de investigación "Comportamiento humano, genética y ambiente", del Centro de Investigación de Procesos Básicos, Metodología y Educación de la Facultad de Psicología, el cual se encuentra aprobado y subsidiado por la Secretaría de Ciencia y Técnica de la Universidad Nacional de Mar del Plata, Argentina. En el marco de convenios firmados con dos instituciones de salud de la ciudad de Mar del Plata, y a partir de los datos proporcionados por el médico derivador, se establecieron los contactos con las madres de niños con TDAH. Por otro lado, se contactó a las madres de niños de la muestra control, los cuales asistían a las mismas instituciones educativas que los niños con TDAH, siendo compañeros de curso de ellos. A cada participante se le administraron las dos escalas (Afecto y Normas-Exigencias) para evaluar estilos educativos parentales (Bersabé et al., 2001) y el Cuestionario de Afrontamiento del Estrés (Sandín y Chorot, 2003).

La participación fue voluntaria y sujeta al consentimiento informado de los participantes. Durante el desarrollo del trabajo se respetaron los principios éticos de la investigación con seres humanos, procurándose las condiciones necesarias para proteger la confidencialidad y actuar sin perjuicio de los participantes. Para la implementación de esta investigación se utilizaron los procedimientos sugeridos por el Programa Temático Interdisciplinario en Bioética de la Universidad Nacional de Mar del Plata, que cuenta con la aprobación del Comité de Ética de dicha institución educativa.

\section{Análisis estadístico}

Para realizar los análisis estadísticos se utilizó el paquete estadístico sPss versión 15. Acorde con los objetivos propuestos, los datos obtenidos fueron sometidos a un análisis estadístico descriptivo. Para la realización de los análisis se tuvieron en cuenta las siguientes variables predictoras en lo respectivo al estilo parental desde la perspectiva de la madre: apoyo, crítica, inductiva, rígida e indulgente. Las variables predictoras relacionadas con las estrategias de afrontamiento fueron: focalizado en la solución del problema (FSP), autofocalización negativa (AFN), reevaluación positiva (REP), expresión emocional abierta (EEA), evitación (EVT), búsqueda de apoyo social (BAS), religión (RLG), afrontamiento racional (AR) y afrontamiento emocional (AE). Con el objeto de determinar si las diferencias entre los dos grupos resultan estadísticamente significativas se aplicó la prueba no paramétrica u de Mann-Whitney, ya que, dado el tamaño de la muestra, esta prueba representa una alternativa a la prueba $t$ sobre diferencia de medias cuando no se cumplen los supuestos en los que se basa la prueba t (normalidad y homocedasticidad; Pardo y Ruiz, 2000). Asimismo, se realizaron análisis de correlaciones mediante la obtención del coeficiente de correlación de Pearson que se utiliza con el fin de medir el grado de relación de dos variables cuantitativas. Adicionalmente, se calculó, para cada una de las variables, el tamaño del efecto de la diferencia, para lo cual se utilizó la clasificación por rangos de Cohen, que indica que el tamaño del efecto puede ser grande (mayor a 0,8 ), mediano (cercano a 0,5 ) o pequeño (menor a 0,2) (Quezada, 2007).

\section{Resultados}

En la tabla 1 se presentan los estadísticos descriptivos de los estilos parentales en función de la pertenencia al grupo de madres de niños con TDAH o al grupo control; los resultados de la prueba u de Mann-Whitney y tamaño del efecto Cohen (D). 
Tabla 1

Estadísticos descriptivos de los estilos parentales discriminados por grupo, resultados de la prueba U-Mann-Whitney y tamaño del efecto Cohen

\begin{tabular}{|c|c|c|c|c|c|c|c|c|c|}
\hline \multicolumn{2}{|c|}{ Estilo materno } & \multicolumn{4}{|c|}{ Madres } & \multirow{3}{*}{$\begin{array}{l}\text { U de Mann- } \\
\text { Whitney }\end{array}$} & \multirow{3}{*}{$\mathbf{z}$} & \multirow{3}{*}{$\begin{array}{l}\text { Sig. Asintót. } \\
\text { (bilateral) }\end{array}$} & \multirow{3}{*}{ d } \\
\hline \multirow[t]{2}{*}{ Escala } & \multirow[t]{2}{*}{ Factores } & \multicolumn{2}{|c|}{$\begin{array}{c}\text { Grupo Control } \\
\text { n } 14\end{array}$} & \multicolumn{2}{|c|}{$\begin{array}{c}\text { Grupo TDAH } \\
\text { n } 35\end{array}$} & & & & \\
\hline & & Media & DE & Media & DE & & & & \\
\hline \multirow{2}{*}{ EA } & Afecto & 45,06 & 4,24 & 43,00 & 6,52 & 196,50 & $-1,07$ & 0,28 & 0,41 \\
\hline & Crítica & 15,29 & 3,36 & 25,50 & 6,60 & 38,00 & $-4,59$ & $0,001^{*}$ & 2,27 \\
\hline \multirow{3}{*}{ ENE } & Inductiva & 43,00 & 4,84 & 39,71 & 5,70 & 160,00 & $-1,88$ & $0,05^{* *}$ & 0,64 \\
\hline & Rígida & 27,71 & 7,94 & 32,21 & 5,72 & 165,00 & $-1,77$ & 0,07 & 0,60 \\
\hline & Indulgente & 14,71 & 3,99 & 17,07 & 5,85 & 197,00 & $-1,06$ & 0,28 & 0,51 \\
\hline
\end{tabular}

Nota. EA: escala de afecto/comunicación; ENE: escala de normas y exigencias. Elaboración propia.

${ }^{\star}$ Diferencias significativas al nivel 0,001. ${ }^{* *}$ Diferencias significativas al nivel 0,05

Tabla 2

Estadísticos descriptivos de las estrategias de afrontamiento discriminados por grupo, resultados de la prueba U-Mann-Whitney y tamano del efecto Cohen

\begin{tabular}{|c|c|c|c|c|c|c|c|c|}
\hline \multirow{3}{*}{$\begin{array}{l}\text { Estrategias de } \\
\text { afrontamiento }\end{array}$} & \multicolumn{4}{|c|}{ Madres } & \multirow{3}{*}{$\begin{array}{l}\text { u de Mann- } \\
\text { Whitney }\end{array}$} & \multirow{3}{*}{$\mathbf{z}$} & \multirow{3}{*}{$\begin{array}{l}\text { Sig. Asintót. } \\
\text { (bilateral) }\end{array}$} & \multirow{3}{*}{ d } \\
\hline & \multicolumn{2}{|c|}{$\begin{array}{c}\text { Grupo control } \\
\text { n } 14\end{array}$} & \multicolumn{2}{|c|}{$\begin{array}{l}\text { Grupo TDAH } \\
\text { n } 35\end{array}$} & & & & \\
\hline & Media & DE & Media & DE & & & & \\
\hline FSP & 15,26 & 5,20 & 17,57 & 4,63 & 182,50 & $-1,38$ & 0,16 & 0,45 \\
\hline AFN & 6,80 & 3,49 & 8,50 & 4,62 & 180,50 & $-1,43$ & 0,15 & 0,44 \\
\hline REP & 16,94 & 4,16 & 15,79 & 3,74 & 197,00 & $-1,06$ & 0,28 & 0,28 \\
\hline EEA & 7,83 & 4,43 & 9,86 & 5,58 & 197,00 & $-1,06$ & 0,28 & 0,42 \\
\hline EVT & 8,91 & 4,82 & 8,29 & 4,84 & 223,50 & $-0,47$ & 0,63 & 0,12 \\
\hline BAS & 11,34 & 7,02 & 14,00 & 5,58 & 184,00 & $-1,35$ & 0,17 & 0,40 \\
\hline RLG & 11,63 & 6,94 & 8,57 & 7,65 & 179,50 & $-1,45$ & 0,14 & 0,42 \\
\hline $\mathrm{AR}$ & 43,54 & 12,38 & 47,36 & 10,73 & 194,50 & $-1,11$ & 0,26 & 0,32 \\
\hline $\mathrm{AE}$ & 14,63 & 6,80 & 18,36 & 7,71 & 171,50 & $-1,63$ & 0,10 & 0,52 \\
\hline
\end{tabular}

Nota. FSP: focalizado en la solución del problema; AFN: autofocalización negativa; REP: reevaluación positiva; EEA: expresión emocional abierta; EVT: evitación; BAS: búsqueda de apoyo social; RLG: religión; AR: afrontamiento racional; AE: afrontamiento emocional. Elaboración propia.

Se puede observar que, si bien se presentan diferencias entre el grupo de madres de niños con TDAH y el grupo control en las dos escalas de afecto/comunicación y normas/exigencias, la prueba confirma diferencias estadísticamente significativas sólo en las subescalas critica/rechazo e inductiva.

En las demás subescalas se observa que el grupo de madres de niños con TDAH presenta valores de percepción inferior en la subescala afecto/comunicación y valores superiores en las subescalas rígida e indulgente, aunque las diferencias no resultan significativas estadísticamente.

En la tabla 2 se presentan los estadísticos descriptivos de las estrategias de afrontamiento en función de la pertenencia al grupo de madres de niños con TDAH o al grupo control, los resultados de la prueba u de MannWhitney y tamaño del efecto Cohen (D).
Se puede observar que el grupo de madres de niños con TDAH presenta valores de percepción inferior en las subescalas reevaluación positiva (REP), evitación (EVT) y principalmente en religión (RLG), y valores superiores en las subescalas focalizado en la solución del problema (FSP), autofocalización negativa (AFN), expresión emocional abierta (EEA), búsqueda de apoyo social (BAS), afrontamiento racional (AR) y principalmente en afrontamiento emocional ( $\mathrm{AE}$ ), aunque las diferencias no resultan estadísticamente significativas.

Por otro lado, se observa que las estrategias más utilizadas, tanto en el grupo de madres de niños con TDAH como en el grupo control, son la estrategia focalizada en la solución del problema (FSP) y la reevaluación positiva (REP), las cuales constituyen la dimensión afrontamiento racional (AR). 
Con el objeto de identificar si existe correlación entre la percepción del estilo parental con las estrategias de afrontamiento en función de los grupos, se realizó un análisis correlacional cuyos resultados se presentan en las tablas 3 y 4 .

Tabla 3

Análisis de correlaciones entre la percepción del estilo parental y las estrategias de afrontamiento en el grupo de madres de niños con TDAH.

\begin{tabular}{|c|c|c|c|c|c|c|c|}
\hline & & & & & stilo materr & & \\
\hline & & $\begin{array}{c}\text { Madres Grupo TDAH } \\
n 14\end{array}$ & & EA & & & \\
\hline & & & Afecto & Crítica & Inductiva & Rígida & Indulgente \\
\hline & FSP & Correlación de Pearson & 0,38 & $-0,38$ & 0,46 & $-0,08$ & $-0,43$ \\
\hline$\stackrel{0}{\stackrel{2}{ }}$ & AFN & Correlación de Pearson & $-0,07$ & 0,37 & $-0,15$ & 0,31 & $-0,17$ \\
\hline$\ddot{\Xi}$ & REP & Correlación de Pearson & $-0,06$ & $-0,23$ & 0,24 & $-0,06$ & 0,03 \\
\hline 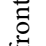 & EEA & Correlación de Pearson & 0,15 & 0,19 & 0,41 & 0,21 & 0,02 \\
\hline 㟧 & EVT & Correlación de Pearson & $-0,38$ & 0,38 & $-0,30$ & 0,41 & 0,37 \\
\hline$\stackrel{\mathscr{a}}{\mathscr{n}}$ & BAS & Correlación de Pearson & 0,25 & $-0,45$ & 0,23 & $-0,08$ & $-0,64^{*}$ \\
\hline 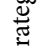 & RLG & Correlación de Pearson & 0,23 & $-0,27$ & 0,24 & $-0,32$ & $-0,05$ \\
\hline 苍 & AR & Correlación de Pearson & 0,27 & $-0,48$ & 0,41 & $-0,10$ & $-0,51$ \\
\hline & $\mathrm{AE}$ & Correlación de Pearson & 0,06 & 0,36 & 0,20 & 0,34 & $-0,08$ \\
\hline
\end{tabular}

Nota. EA: escala de afecto/comunicación; ENE: escala de normas y exigencias; FSP: focalizado en la solución del problema; AFN: autofocalización negativa; REP: reevaluación positiva; EEA: expresión emocional abierta; EVT: evitación; BAs: búsqueda de apoyo social; RLG: religión; AR: afrontamiento racional; AE: afrontamiento emocional. Elaboración propia.

${ }^{*}$ La correlación es significativa al nivel 0,01.

Se puede observar que en el grupo de madres de niños con TDAH se presenta correlación negativa entre la subescala de estilo parental indulgente y la estrategia de afrontamiento búsqueda de apoyo social (BAs), no presentándose correlaciones a nivel significativo entre las demás subescalas de estilos parentales y afrontamiento al estrés.

Tabla 4

Análisis de correlaciones entre la percepción del estilo parental y las estrategias de afrontamiento en el grupo control

\begin{tabular}{|c|c|c|c|c|c|c|c|}
\hline & & \multirow{3}{*}{$\begin{array}{l}\text { Madres grupo control } \\
\text { n } 35\end{array}$} & \multicolumn{5}{|c|}{ Estilo parental } \\
\hline & & & \multicolumn{3}{|c|}{ EA } & \multicolumn{2}{|c|}{ ENE } \\
\hline & & & Afecto & Crítica & Inductiva & Rígida & Indulgente \\
\hline \multirow{9}{*}{ 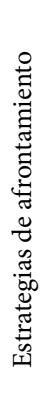 } & FSP & Correlación de Pearson & 0,09 & $-0,15$ & 0,08 & 0,21 & $-0,10$ \\
\hline & AFN & Correlación de Pearson & $-0,13$ & 0,17 & $-0,07$ & 0,12 & 0,31 \\
\hline & REP & Correlación de Pearson & $0,36^{*}$ & $-0,48^{* *}$ & 0,23 & $-0,14$ & 0,04 \\
\hline & EEA & Correlación de Pearson & $-0,27$ & 0,23 & 0,11 & $0,36^{*}$ & $-0,13$ \\
\hline & EVT & Correlación de Pearson & 0,28 & $-0,25$ & $-0,12$ & 0,01 & $0,33^{*}$ \\
\hline & BAS & Correlación de Pearson & $-0,04$ & $-0,07$ & $-0,13$ & $-0,20$ & 0,07 \\
\hline & RLG & Correlación de Pearson & 0,12 & $-0,41^{*}$ & 0,02 & $-0,25$ & $-0,06$ \\
\hline & AR & Correlación de Pearson & 0,13 & $-0,27$ & 0,03 & $-0,07$ & 0,01 \\
\hline & $\mathrm{AE}$ & Correlación de Pearson & $-0,24$ & 0,23 & 0,03 & 0,30 & 0,07 \\
\hline
\end{tabular}

Nota. EA: escala de afecto/comunicación; ENE: escala de normas y exigencias; FSP: focalizado en la solución del problema; AFN: autofocalización negativa; REP: reevaluación positiva; EEA: expresión emocional abierta; EVT: evitación; BAS: búsqueda de apoyo social; RLG: religión; AR: afrontamiento racional; AE: afrontamiento emocional. Elaboración propia.

${ }^{*}$ La correlación es significante al nivel 0,05

${ }^{*}$ La correlación es significativa al nivel 0,01 
Se puede observar que en el grupo control se presentan correlaciones positivas entre la subescala de estilo parental afecto y la estrategia de afrontamiento reevaluación positiva (REP), entre la subescala de estilo parental rígida y la estrategia de afrontamiento expresión emocional abierta (EEA) y entre la subescala de estilo parental indulgente y la estrategia de afrontamiento evitación (ЕVT). Asimismo, se presentan correlaciones negativas entre la subescala de estilo parental crítica y la estrategia de afrontamiento reevaluación positiva (REP), y entre la subescala de estilo parental crítica y la estrategia de afrontamiento religión (RLG).

\section{Discusión}

El objetivo general del presente trabajo fue analizar y comparar la percepción de las estrategias de afrontamiento y el estilo parental de madres de niños con y sin diagnóstico de trastorno con déficit de atención e hiperactividad (TDAH). Los resultados obtenidos muestran diferencias en la percepción de los estilos parentales de las madres de niños con TDAH en comparación con el grupo control. Si bien presentan un perfil de estilo parental con características dentro de los perfiles poblacionales esperados, sí existen diferencias significativas entre las dos muestras estudiadas, lo cual podría indicar un estilo parental diferencial y característico de las familias de niños con TDAH.

Se evidencia que la percepción del estilo parental de las madres de niños con TDAH se basa en una tendencia a manifestar mayor crítica y rechazo, acompañada de una menor expresión afectiva, aceptación y comunicación, por lo que las madres de niños con TDAH manifestarían mayor crítica hacia sus hijos, menor aceptación y confianza, falta de apoyo y sostén, con dificultades en la comunicación. Lo anterior está en concordancia con los patrones de interacción entre las madres y sus hijos con TDAH descritos por la literatura científica, caracterizados por madres que responden menos a las interacciones iniciadas por sus hijos, con un estilo de respuesta negativa-reactiva, impositiva, controladora y con menos estrategias de parentalidad positiva; además de menor afectuosidad y con un estilo educativo más negligente (García-Quiroga e IbáñezFanes, 2007; Goldstein et al., 2007; Santurde del Arco y Del Barrio del Campo, 2010; Gau y Chang, 2013).

En lo que respecta a las normas y exigencias, se evidenció que las madres de niños con TDAH perciben su estilo educativo menos inductivo o democráti- co que las madres de niños sin el trastorno, por lo que tenderían a ser más rígidas, con una predisposición a mantener un nivel de exigencias alto e inadecuado a las necesidades de los hijos, y a imponerles el cumplimiento de las normas y los límites, aunque de maneras no consistentes ni claras. En este sentido, Bersabé et al. (2001) sostienen que cuando estas madres establecen los límites, con regularidad no exigen su cumplimiento, y al mismo tiempo imponen dicho cumplimiento desde la crítica y no tendientes a un estilo más democrático que implicaría establecer las normas desde el afecto, el interés y la comunicación.

De la misma manera, las madres de niños con TDAH suelen percibir la relación con sus hijos como significativamente más negativa que las madres de niños que no presentan este trastorno, percibiéndose a sí mismas menos competentes y con una satisfacción parental más baja (Barkley, 1990; Santurde del Arco y Del Barrio del Campo, 2010).

En cuanto a las estrategias de afrontamiento al estrés utilizadas, se observa que no se presentan diferencias significativas entre el grupo de madres de niños con TDAH y el grupo control. Esto concuerda con estudios anteriores en los que se sostiene que a pesar de las diferencias en los niveles de estrés, ambos grupos utilizan similares cantidades y tipos de estrategias de afrontamiento (López et al., 2008, Tajalli et al., 2011).

Sin embargo, se puede observar que las madres de niños con diagnóstico de TDAH presentan una tendencia a utilizar en mayor medida las siguientes estrategias: focalizado en la solución del problema (FSP), autofocalización negativa (AFN), expresión emocional abierta (EEA) y búsqueda de apoyo social (BAS). Asimismo, utilizan en mayor medida las estrategias de afrontamiento generales: racional (AR) y emocional (AE).

Precisamente, en estudios previos se demostró que, frente a las dificultades crónicas de los hijos, la mayoría de las madres movilizan esfuerzos para hacer frente a la situación, es decir, utilizan estrategias de afrontamiento activas, racionales o focalizadas en el problema, otorgándole un nuevo significado al problema, intentando extraer lo positivo de la crisis con el objetivo de modificar la situación (Loubat y Cuturrufo, 2007; Wang, Michaels y Day, 2011).

Si bien ya se ha evidenciado que en general las mujeres buscan frecuentemente más apoyo social para resolver sus problemas compartiéndolos con otros e intentan analizar sistemáticamente diversos puntos de vista o soluciones en mayor medida que los hombres, se observa que las madres de niños con TDAH hacen 
más uso de estas estrategias que las madres de niños sin el trastorno. Justamente, esta estrategia de buscar soporte en personas e instituciones cuando se experimentan situaciones de tensión se ha considerado una forma positiva y adaptativa de afrontar los problemas, ya que implica el manejo directo de estos por medio de la orientación que otros puedan proveer, disminuyendo la tendencia a huir o a evitar (De la Huerta, Corona y Méndez, 2006). Este estilo de afrontamiento activo les permite a las madres buscar información y solucionar los problemas, por ejemplo, acercándose a un profesional, tratando de saber más sobre el trastorno, buscando apoyo familiar y estableciendo planes de acción para el cuidado de sus hijos, lo cual permite reducir los niveles de estrés parental (Tajalli et al., 2011).

También se observa que estas madres tienden a utilizar estrategias de afrontamiento emocional, en las que se encuentran la utilización de la expresión emocional abierta (EEA) y la autofocalización negativa (AFN). En este sentido, las madres de niños con TDAH tenderían a descargar el mal humor con los demás, comportándose hostilmente para desahogar su emocionalidad, y también tenderían a autoconvencerse negativamente, autoinculparse hasta llegar a resignarse, asumiendo su propia incapacidad para resolver la situación (Sandín y Chorot, 2003). Esto se corrobora con lo hallado en un estudio realizado por Durkan et al. (2008) en el que se evidencia una mayor tendencia a utilizar estrategias de expresión emocional, negación, conductas afrontativas disfuncionales, junto con estrategias de focalización en el problema.

Así, si bien estas madres tienden a la resolución del problema, presentan episodios de desborde emocional, precisamente porque la crianza de un niño con TDAH ofrece numerosos retos debido a que ellos poseen más dificultades para obedecer las órdenes e inhibir las respuestas impulsivas y son menos sensibles a las peticiones de los padres, por lo que su estilo personal desencadena cogniciones negativas sobre el rol de padres, baja autoestima y sentimientos de culpa (Grau-Sevilla, 2007; Alizadeh et al., 2007).

Por otro lado, si bien se observa una tendencia a utilizar estrategias centradas en la emoción, lo cual tiende a ser disfuncional, presentan baja utilización de estrategias de evitación, es decir, no se concentran en otras cosas, como inclinarse al trabajo o a otras actividades con el fin de no pensar en el problema (Sandín y Chorot, 2003).

En síntesis, las madres de niños con TDAH utilizan menos estrategias de evitación y más estrategias focalizadas en la resolución de problemas y búsqueda de apoyo social, lo cual también se ve reflejado en una tendencia a presentar un estilo educativo menos indulgente y permisivo. Así, estas madres no tienden a evitar la puesta de límites, sino, por el contrario, intentan que sus hijos cumplan las normas y buscan maneras de ir solucionando los problemas. Esto se evidencia precisamente en la relación encontrada entre el estilo parental indulgente o permisivo y el menor uso de estrategias de afrontamiento tendiente a la búsqueda de apoyo social.

Por último, los resultados muestran diversas relaciones consistentes entre los distintos estilos parentales y ciertas estrategias de afrontamiento, especialmente en la muestra control. Esto podría abrir el debate y el desarrollo de futuros estudios tendientes a indagar la posible dependencia de ambos constructos ya que a nivel empírico se encuentran muy relacionados y podrían ser aspectos de un mismo fenómeno. Si bien no se evidencia una gran cantidad de estudios previos en los que se hayan vinculado ambas variables, el estudio realizado por Henao-López (2008) podría dar respaldo a las relaciones encontradas entre los constructos, considerando como hipótesis teórica que las estrategias de afrontamiento parentales forman parte del perfil cognitivo personal de los padres (Beck, Wrigth, Newman y Liese, 1993), y que al consistir en actitudes, comportamientos y estrategias personales (Clark y Beck, 1997), pueden solaparse, o incluso ser parte del estilo parental, es decir, de las actitudes, las creencias y los comportamientos a la hora de relacionarse con el hijo. Por ello, será de interés profundizar el análisis en esta línea de investigación.

Una de las limitaciones del estudio es el tamaño de los grupos, y esto se debe a los inconvenientes que se presentan habitualmente para evaluar pacientes diagnosticados con alguna patología y que cumplan con todos los criterios de inclusión que se requieren en un diseño de investigación. Por esto, sería recomendable para futuros estudios ampliar la muestra a un número mayor de participantes, diferenciando distintos grupos de niños con TDAH en relación con los subtipos y las comorbilidades, y también incluir en el análisis variables contextuales como el estilo parental del padre, el tipo de familia, los conflictos de pareja, el número de hermanos, la afectación de alguno(s) de los hermanos, las creencias acerca del trastorno y respecto a los hijos, entre otras.

De todas maneras, los datos aportados pueden constituir una base para el diseño de estudios que profundicen el análisis y posibiliten la caracterización de 
estilos parentales educativos en familias que tengan hijos con TDAH. En la práctica clínica los resultados constituyen un aporte para la intervención con dichas familias, al resaltar la importancia de favorecer el desarrollo de técnicas efectivas de disciplina y una adecuada comunicación y afecto en la relación de las madres con sus hijos; asimismo, proporcionarle a estas madres recursos en materia de estrategias de afrontamiento que amortigüen los efectos estresantes de la crianza y convivencia con niños que presentan dicho trastorno (Martínez, 2010; Healey, Flory, Miller y Halperin, 2011; Figueroa, Aburto y Acevedo, 2012). Incrementar el conocimiento acerca de cuáles son aquellas estrategias de afrontamiento al estrés más efectivas, $\mathrm{y}$ bajo qué circunstancias pueden ser más beneficiosas para estas madres, tiene una implicación directa sobre el tratamiento del trastorno (Lyons, Leon, Phelps y Dunleavy, 2010), precisamente porque las estrategias y los estilos de afrontamiento que utilicen las madres frente a las situaciones de crisis podrían jugar un rol importante en el curso de la enfermedad de su hijo.

\section{Referencias}

Alizadeh, H., Applequist, K. F. y Coolidge, F. L. (2007). Parental Self-Confidence, Parenting Styles, and Corporal Punishment in Families of ADHD Children in Iran. Child Abuse y Neglect, 31(5), 567-572. doi:10.1016/j. chiabu.2006.12.005

Alizadeh, H. y Andries, C. (2002). Interaction of Parenting Styles and Attention Deficit Hyperactivity Disorder in Iranian Parents. Child and Family Behavior Therapy, 24(3), 37-52. doi: 10.1300/J019v24n03_03

American Psychiatric Association (APA). (2002). Manual Diagnóstico y Estadístico de los Trastornos Mentales DSM-IV-TR. Barcelona: Masson.

Barkley, R. A. (1990). Attention Deficit Hyperactivitv Disorder: A handbook for diagnosis and treatment. New York: Guilford Publications.

Baumrind, D. (1996). The discipline controversy revisited. Family Relations, 45(4), 405-414. Recuperado de http:// www.jstor.org/stable/585170

Baumrind, D., Larzelere, R. E. y Owens, E. B. (2010). Effects of Preschool Parents Power Assertive Patterns and Practices on Adolescent Development. Parenting: Science and Practice, 10(3), 157-201. doi: 10.1080/15295190903290790

Beck, A., Wrigth, F. W., Newman, C. F. y Liese, B. (1993). Cognitive therapy of substance abuse. New York: Guilford Press.
Bersabé, R., Fuentes, M. J. y Motrico, E. (2001). Análisis psicométrico de dos escalas para evaluar estilos educativos parentales. Psicothema, 13(4), 678-684. Recuperado de http://156.35.33.98/reunido/index.php/PST/article/ viewFile/7885/7749

Billings, A. G. y Moos, R. H. (1981). The Role of Coping Responses and Social Resources in Attenuating the Stress of Life Events. Journal of Behavioral Medicine, 4(2), 139157. doi: 10.1007/BF00844267

Cardo, E. y Servera M. (2008). Trastorno por déficit de atención/hiperactividad: estado de la cuestión y futuras líneas de investigación. Revista de Neurología, 46(6), 365-72. Recuperado de http://faros.hsjdbcn.org/ adjuntos/130.2-tdha.pdf

Catelan-Mainardes, S. C. (2010). Transtorno de déficit de atenção e hiperatividade na infância e adolescência pela perspectiva da neurobiologia. Revista Saúde e Pesqui$s a, 3(3), 385-391$. Recuperado de http://www.cesumar. br/pesquisa/periodicos/index.php/saudpesq/issue/ view/75

Clark, D. A. y Beck, A. (1997). El estado de la cuestión en la teoría y la terapia cognitiva. En I. Caro (Ed.), Manual de psicoterapias cognitivas. Barcelona: Paidós.

Cockerham, W. C. (2001). Handbook of Medical Sociology. Nueva York: Prentice-Hall.

Cussen A., Sciberras E., Ukoumunne, O. C. y Efron D. (2012). Relationship between Symptoms of AttentionDeficit/Hyperactivity Disorder and Family Functioning: A Community-Based Study. European Journal of Pediatrics, 171(2), 271-280. doi: 10.1007/s00431011-1524-4

Dabrowska, A. y Pisula, E. (2010). Parenting Stress and Coping Styles in Mothers and Fathers of Pre-School Children with Autism and Down Syndrome. Journal of Intellectual Disability Research, 54(3), 266-280. doi: 10.1111/j.1365-2788.2010.01258.x

De la Huerta, R., Corona, J. y Méndez, J. (2006). Evaluación de los estilos de afrontamiento en cuidadores primarios de niños con cáncer. Revista Neurología, Neurocirugía y Psiquiatría, 39(1), 46-51. Recuperado de http:// www.medigraphic.com/pdfs/revneuneupsi/nnp-2006/ nnp061g.pdf

Durkan, I., Erdem, M. y Tufan, E. (2008). Depression and Anxiety Levels and Coping Strategies Used by Mothers of Children with adhd: A Preliminary Study. Anatolian Journal of Psychiatry, 9, 217-223.

Etchepareborda, M. C., Díaz-Lucero, A. y de Ramón, I. (2011). Diagnóstico del TDAH. En M. C. Etchepareborda (Ed.), TDAH + FE: trastorno por déficit de atención con hiperactividad y de las funciones ejecutivas: abordaje interdisciplinar (pp. 37-43). Buenos Aires: Rivolin. 
Figueroa, A. J., Aburto, M. A. C. y Acevedo, R. Z. (2012). Conflicto trabajo-familia, autoeficacia parental y estilos parentales percibidos en padres y madres de la ciudad de Talca, Chile. Acta Colombiana de Psicología, 15(1), 57-65. Recuperado de http://repository.ucatolica.edu. co/bitstream/10983/697/1/v15n1a06.pdf

Folkman, S. y Lazarus, R. S. (1988). Coping as a Mediator of Emotion. Journal of Personality and Social Psychology, 54(3), 466-475. doi: 10.1037/0022-3514.54.3.466

García-Quiroga, M. e Ibáñez-Fanes, M. (2007). Apego e hiperactividad: Un estudio exploratorio del vínculo madre-hijo. Terapia Psicológica, 25(2), 123-134. doi: 10.4067/S0718-48082007000200003

Gau, S. S. F. y Chang, J. P. C. (2013). Maternal parenting styles and mother-child relationship among adolescents with and without persistent attention-deficit/hyperactivity disorder. Research in Developmental Disabilities, 34(5), 1581-1594. doi: 10.1016/j.ridd.2013.02.002

Ghuman, J. y Ghuman, H. (2014). adhd in Preschool Children: Overview and Diagnostic Consideration. En J. Ghuman y H. Ghuman (Eds.), adhd in Preschool Children: Assessment and Treatment. New York: Oxford University Press.

Goldstein, L. H., Harvey, E. A., y Friedman-Weieneth, J. L. (2007). Examining Subtypes of Behavior Problems Among 3-Year-Old Children, Part III: Investigating Differences in Parenting Practices and Parenting Stress. Journal of Abnormal Child Psychology, 35(1), 125-136. doi: 10.1007/s10802-006-9047-6

Grañana, N.; Richaudeau, A., Robles, C., Scotti, M. E., Fejerman, N. y Allegri, R. (2006). Detección de síntomas para trastorno por déficit de atención e hiperactividad: Escala SNAP IV Validación en Argentina. Revista Neurológica Argentina, 28(1), 20. Recuperado de http://www. sna.org.ar/images/revista/2006/supl/poster1.pdf

Graziano, P. A., McNamara, J. P., Geffken, G. R. y Reid, A. (2011). Severity of Children's adhd Symptoms and Parenting Stress: A Multiple Mediation Model of Self-Regulation. Journal of Abnormal Child Psychology, 39(7), 1073-1083. doi: 10.1007/s10802-011-9528-0

Grau-Sevilla, M. D. (2007). Análisis del contexto familiar en niños con TDAH (Tesis doctoral). Universidad de Valencia, España.

Healey, D. M., Flory, J. D., Miller, C. J. y Halperin, J. M. (2011). Maternal Positive Parenting Style is Associated with Better Functioning in Hyperactive/Inattentive Preschool Children. Infant and Child Development, 20(2), 148-161. doi: 10.1002/icd.682

Henao-López, G. C. (2008). Perfil cognitivo parental (esquemas maladaptativos y estrategias de afrontamiento), estilo de interacción parental y su influencia en el desarrollo emocional infantil. Recuperado de http://bibliotecavirtual.clacso.org.ar/Colombia/alianza-cindeumz/20091119041143/tesis-henao.pdf

Lazarus, R. S. (1993). Coping Theory and Research: Past, Present, and Future. Psychosomatik Medicine, 55, 234-247.

Lazarus, R. S. y Folkman, S. (1984). Stress, Appraisal and Coping. New York: Springer.

Long, C., Gurka, M. y Blackman, J. (2008). Family Stress and Children's Language and Behavior Problems: Results from the National Survey of Children's Health. Topics in Early Childhood Special Education, 28(3), 148-157. doi: $10.1177 / 0271121408318678$

López-Martín, S., Albert, J., Fernández-Jaén, A. y Carretié, L. (2010). Neurociencia afectiva del tDAH: datos existentes y direcciones futuras. Escritos de Psicología, 3(2), 17-29. Recuperado de http://scielo.isciii.es/scielo. php?pid=S1989-38092010000100003yscript=sci_arttext

López-Soler, C., Puerto, J. C., López-Pina, J. A. y Prieto, M. (2009). Percepción de los estilos educativos parentales e inadaptación en menores pediátricos. Anales de Psicología, 25(1), 70-77. Recuperado de file://C:/Users/ usuario/Downloads/71511-296821-1-PB.pdf

Lopez, V., Clifford, T., Minnes, P. y Ouellette-Kuntz, H. (2008). Parental Stress and Coping in Families of Children with and without Developmental Delays. Journal on Developmental Disabilities, 14(2), 99-104. Recuperado de http://www.oadd.org/Journal_14.html.

Loubat, M. y Cuturrufo A. N. (2007). Estrategias de afrontamiento de padres respecto de los primeros brotes psicóticos de sus hijos y su relación con la adhesión a tratamiento. Revista Chilena de Neuro-Psiquiatría, 45(4), 269-277. doi: 10.4067/S0717-92272007000400002

Lyons, A. M., Leon, S. C., Phelps, C. E. R. y Dunleavy, A. M. (2010). The Impact of Child Symptom Severity on Stress Among Parents of Children with AsD: The Moderating Role of Coping Styles. Journal of Child and Family Studies, 19(4), 516-524. doi: 10.1007/s10826009-9323-5

Martínez, Á. C. (2010). Pautas de crianza y desarrollo socioafectivo en la infancia. Diversitas, 6(1), 111-121.

McKee, T. E., Harvey, E., Danforth, J. S., Ulaszek, W. R. y Friedman, J. L. (2004). The Relation between Parental Coping Styles and Parent-Child Interactions before and after Treatment for Children with adhd and Oppositional Behavior. Journal of Clinical Child and Adolescent Psychology, 33(1), 158-168. doi: 10.1207/S15374424JCCP3301_15

Mikulic, I. M. y Crespi, M. C. (2008). Adaptación y validación del inventario de respuesta de afrontamiento de Moss (CRI-A) para adultos. Anuario de Investigaciones, 15(2), 305-312. 
Miranda, A., Presentación, M. J., Colomer, C. y Roselló, B. (2011). Satisfacción con la vida de niños con trastorno por déficit de atención/hiperactividad: estudio de posibles factores de riesgo y de protección. Revista de Neurología, 52(1), 119-126. Recuperado de http://repositori.uji.es/xmlui/bitstream/handle/10234/38420/47873. pdf? sequence $=1$

Miranda-Casas, A., Grau-Sevilla, D., Marco-Taberner, R. y Roselló, B. (2007). Estilos de disciplina en familias con hijos con trastorno por déficit de atención/hiperactividad: influencia en la evolución del trastorno. Revista de Neurología, 44(2), 23-25. Recuperado de http://neuroser.com/ pdfs/Estilos\%20de\%20disciplina\%20en\%20familias\%20 con\%20hijos\%20con\%20tdah,\%20influencia\%20en\%20 la\%20evoluci\%F3n\%20del\%20trastorno..pdf

Montero, I. y León, O. (2007). A Guide for Naming Research Studies in Psychology. International Journal of Clinical and Health Psychology, 7(3), 847-862. Recuperado de http://aepc.es/ijchp/gneip07_es.pdf

Montiel-Nava, C., Montiel-Barbero, I. y Peña, J. A. (2005). Clima familiar en el trastorno por déficit de atención-hiperactividad. Psicología Conductual, 13(2), 297-310. Recuperado de http://fundacioncadah.org/ j289eghfd7511986_uploads/20120606_H9wFZ9h2YftQL84peS4H_0.pdf

Narkunam, N., Hashim, A. H., Sachdev, M. K., Pillai, S. K. y Ng, C. G. (2014). Stress among Parents of Children with Attention Deficit Hyperactivity Disorder, a Malaysian Experience. Asia-Pacific Psychiatry, 6(2), 207-216. doi: 10.1111/j.1758-5872.2012.00216.x

Olhaberry, M. y Farkas, C. (2012). Estrés materno y configuración familiar: Estudio comparativo en familias chilenas monoparentales y nucleares de bajos ingresos. Universitas Psychologica, 11(4), 1317-1326. Recuperado de http://revistas.javeriana.edu.co/index.php/revPsycho/article/view/1317/3360

Olson, D. H. y McCubbin, H. I. (1989). Families: What Makes them Work. California: Sage.

Ortiz-Léon, S. y Jaimes-Medrano, A. L. (2007). El trastorno por déficit de atención e hiperactividad en estudiantes universitarios. Revista de la Facultad de Medicina de la unam, 50(3), 125-127. Recuperado de http://www.medigraphic.com/pdfs/facmed/un-2007/un073g.pdf

Pardo, A. y Ruiz, M. (2000). Spss 11 guía para el análisis de datos. Análisis no paramétrico. México: McGraw-Hill.

Park, H. y Walton-Moss, B. (2012). Parenting Style, Parenting Stress, and Children's Health-Related Behaviors. Journal of Developmental y Behavioral Pediatrics, 33(6), 495-503. doi: 10.1097/DBP.0b013e318258bdb8

Pimentel, M. J., Vieira-Santos, S., Santos, V. y Vale, M. C. (2011). Mothers of Children with Attention Deficit/
Hyperactivity Disorder: Relationship among Parenting Stress, Parental Practices and Child Behaviour. ADHD Attention Deficit and Hyperactivity Disorders, 3(1), 618. doi: 10.1007/s12402-011-0053-3

Quezada, C. (2007). Potencia estadística, sensibilidad y tamaño de efecto: ¿Un nuevo canon para la investigación? Onomázein, 16, 159-170. Recuperado de http://www. onomazein.net/16/potencia.pdf

Raya-Trenas, A. F., Herreruzo-Cabrera, J. y Pino-Osuna, M. J. (2008). El estilo de crianza parental y su relación con la hiperactividad. Psicothema, 20(4), 691-696.

Richaud, M. C. (2005). Estilos parentales y estrategias de afrontamiento en niños. Revista Latinoamericana de Psicología, 37(1), 47-58.

Roselló, B., García-Castellar, R., Tárraga-Mínguez, R. y Mulas, F. (2003). El papel de los padres en el desarrollo y aprendizaje de los niños con trastorno por déficit de atención con hiperactividad. Revista de Neurología, 36(1), 79-S84. Recuperado de http://roderic.uv.es/ jspui/bitstream/10550/32626/1/000729.pdf

Russel, A., Hart, C. H., Robinson, C. C. y Olsen, S. F. (2003). Children's Sociable and Aggressive Behavior with Peers: A Comparison of the us and Australia and Contributions of Temperament and Parenting Styles. International Journal of Behavioral Development, 23, 74-86.

Sadek, J. (2014). Epidemiology and etiology of ADHD. En J. Sadek (Ed.), A Clinician's Guide to adhd (pp. 7-11). Springer International Publishing. doi: 10.1007/978-3319-02359-5_3

Samaniego, V. (1998). El Child Behaviour Checklist: su estandarización y aplicación en un estudio epidemiológico. Problemas comportamentales y sucesos de vida en niños de 6 a 11 años. Buenos Aires: Informe Final ubacyt Mimeo.

Sandín, B. (2003). El estrés: un análisis basado en el papel de los factores sociales. Revista Internacional de Psicología ClínicaydelaSalud, 3(1), 141-157. Recuperado dehttp://osteopatia-moratalaz.es/El\%20estr\%C3\%83\%C2\%A9s\%20 Un\%20an\%C3\%83\%C2\%A1lisis\%20basado\%20en\%20 el\%20papel\%20de\%20los\%20factores\%20sociales.pdf

Sandín, B. y Chorot, P. (2003). Cuestionario de Afrontamiento del Estrés (CAE): Desarrollo y validación preliminar. Revista de Psicopatología y Psicología Clínica, 8(1), 39-54. Recuperado de http://e-spacio.uned.es/revistasuned/index.php/RPPC/article/viewFile/3941/3796

Santurde del Arco, E. y Del Barrio del Campo, J. A. (2010, 9 de febrero). Los modelos de apego y los estilos educativos en niños con trastorno por déficit de atención con hiperactividad. Recuperado de http://www.interpsiquis.com

Siegel, C. y Keefe, C. (2007). Stress and Coping among Parents of Adolescents ADHD (Convention Presentation). American Psychological Association. 
Solem, M. B., Christophersen, K. A. y Martinussen, M. (2011). Predicting Parenting Stress: Children's Behavioural Problems and Parents' Coping. Infant and Child Development, 20(2), 162-180. doi: 10.1002/icd.681

Tajalli, P., Hooman, H. A., Afrooz, G. A. y Bonab, B. G. (2011). A Comparison of Coping Strategies Used by Mothers of Children with/without ADHD. International Journal of Fundamental Psychology and Social Sciences, 1(1), 1113. Recuperado de http://www.fundamentaljournals. org/ijfpss/downloads/3-Tajalli\%20N1\%20p(11-13).pdf

Tan, T. X., Camras, L. A., Deng, H., Zhang, M. y Lu, Z. (2012). Family Stress, Parenting Styles, and Behavioral Adjustment in Preschool-Age Adopted Chinese Girls. Early Childhood Research Quarterly, 27(1), 128-136. http://dx.doi.org/10.1016/j.ecresq.2011.04.002

Tomanik, S., Harris, G. E. y Hawkins, J. (2004). The Relationship between Behaviours Exhibited by Children with Autism and Maternal Stress. Journal of Intellectual and Developmental Disability, 29(1), 6-26, doi: 10.1080/13668250410001662892

Torío-López, S., Peña-Calvo, J. V. y Rodríguez-Menéndez, M. C. (2008). Estilos educativos parentales. Revisión bibliográfica y reformulación teórica, teoría de la educación. Revista Interuniversitaria, 20, 151-178. Recuperado de http://gredos.usal.es/jspui/bitstream/10366/71805/1/ Estilos_educativos_parentales_revision_b.pdf

Verduzco-Alvarez-Icaza, M. A., Lucio-Gómez-Maqueo, E. y Durán-Patiño, C. (2004). La influencia de la autoestima en la percepción del estrés y el afrontamiento en niños de edad escolar. Salud Mental, 27(4), 18-25. Recuperado de http://www.medigraphic.com/pdfs/salmen/sam2004/sam044c.pdf

Wang, P.; Michaels, C. A. y Day, M. S. (2011). Stresses and Coping Strategies of Chinese Families with Children with Autism and Other Developmental Disabilities. Journal of Autism and Developmental Disorders, 41(6), 783-795. doi: 10.1007/s10803-010-1099-3

Wechsler, D. (1994). Test de inteligencia para Niños WISC III. Manual. Buenos Aires: Paidos.

Yousefia, S., Far, A. S. y Abdolahian, E. (2011). Parenting Stress and Parenting Styles in Mothers of ADHD with Mothers of Normal Children. Procedia Social and Behavioral Sciences, 30, 1666-1671. doi: 10.1016/j.sbspro.2011.10.323 